\title{
Mach-Zehnder Interferometer Sensor for Acoustic Detection with Optimal Performance
}

\author{
${ }^{1}$ Prashil M. Junghare, ${ }^{2}$ Dr. Santosh Kulkarni, ${ }^{3}$ Raghavendra Janawad \\ Department of electronics and communication \\ ${ }^{1,2,3}$ K.S. School of Engineering and Management, Bangalore, India
}

\begin{abstract}
This paper investigates analytically the performance of MZI sensor for acoustic detection in terms of light power, fiber characteristic and detectable acoustically/induced phase in the terms of output current at the photomultiplier tube (PMT). In this paper, pressure of $4.7 * 10^{-11}$ Pascal shows the change in the effective length 0.15 meter of fiber and change in the phase of the light path is $6.5 * 10^{-4}$ Radian and output current has been observed for borosilicate crown glass is $1.68 * 10^{\wedge}-19$ Ampere at pressure of $2.5 \mathrm{Mpa}$.
\end{abstract}

Keywords - MZI, Mandrel, Sensitivity, PMT

\section{Introduction}

Mach-Zhender interferometer is a device used to determine the phase shift between two arm-sensor arm \& reference arm) from a coherent light source. It using light modulation technique, to measure small phase shift in one of the two arm caused by a small change in length of one of the paths. Interferometric fiber optic sensor exploits the changes in an optical path length induced by transverse load in the optical fibers. MZI sensors modulate the phase of the electromagnetic waves propagating within the optical waveguide. One of the major areas of application for MZI is in defense. There are two main approaches to coupling a load induced phase shift in an optical fiber to the detection process; a mandrel sensor or a coated fiber sensor ${ }^{[1,2 \text {, and } 3]}{ }^{3}$. Both of these approaches are viable up to $\sim 50 \mathrm{kHz}$. In coated fiber sensors the optical fiber is wound in a coil as a sensing element and the size is comparatively large. Since the optic coupling of the fiber waveguide is weak, a long fiber is generally used to increase the induced phase change.. Mandrel sensors appear to be ideal for point sensors even though they have somewhat limited geometrical versatility.

\section{Pressure Sensitivity Of Mzi}

In a fiber wound mandrel sensor, a thin jacket fiber is typically wrapped around a sensing mandrel and a thin polymer, less than $1 \mathrm{~mm}$ thick, is coated over the fiber as protection during operation. The optical fiber measures the pressure-induced strain in the mandrel and protecting layer. Mandrel sensors are important because they are easy to produce and they exhibit a high sensitivity and amenability to spatial shading. Consider the mandrel sensor shown in Fig. 1(b). A homogeneous cylindrical mandrel of length $L_{m}$ and radius $R_{m}$ is radially wrapped with a single-mode fiber of length $l$ over a length $\mathrm{L}$.

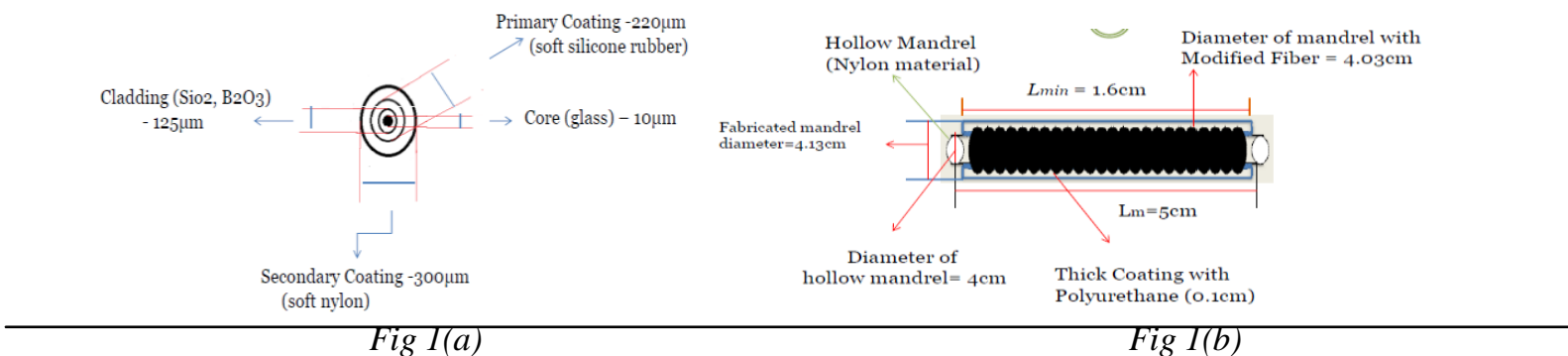

The molding layer is assumed to be only coated on the fiber layer and has a thickness of less than $1 \mathrm{~mm}$. Assuming that the fiber sensor response is driven solely by the mandrel response, it is clear that a Circumferential change in the mandrel couples directly to a length change in the fiber. The phase of light propagating through an optical fiber is defined as,

where $\Phi=\beta * l=n * k_{0} * l$ $\beta$

gation constant in the fiber length direction, 1 is the total fiber length wrapped around the mandrel equal to, $\mathrm{N}$ - is the number of fiber windings round the mandrel, $\mathrm{R}$ is the distance from the center of the mandrel to that of the fiber as denoted in Fig.1 (b), $\mathrm{n}$ is the refractive index of the fiber core, and $\mathrm{k}_{0}$ is the free space propagation constant. The change in the optical phase of the fiber may be written as, 


$$
\Delta \Phi=\beta * \Delta l+\frac{d \beta}{d n} * \Delta n l+\frac{d \beta}{d D} * \Delta D * l
$$

Where, $\mathrm{D}$ is the fiber diameter ${ }^{[5,6]}$. The change in the fiber length using the cylindrical coordinate system is written as,

$$
\Delta l=l *\left(\varepsilon_{r}+\varepsilon_{z}\right)
$$

Where $\square \mathrm{r}$ is the radial strain and $\square \mathrm{z}$ is the axial strain in the fiber with the axis convention with respect to the mandrel in Fig.1. Since the refractive indices of the core and cladding differ only slightly, one can use the approximation of

$$
\frac{d \beta}{d n} \approx k_{0} \ldots \ldots . . .(4)
$$

The change in the refractive index $\Delta \mathrm{n}$ can be written in terms of the change in the optical indicatrix given by the well-known expression ${ }^{[7,8]}$

$$
\Delta\left(\frac{1}{n^{2}}\right)_{i}=\sum_{j=1}^{3} P_{i j} \varepsilon_{j}(i, j=1,2,3) .
$$

Where $p_{i j}$ are elements of the strain optic tensor and $\epsilon_{j}$ are principal strains. Since fluid pressure is transmitted to the transducer only at right angles to its boundary at every point, the components containing a shear strain have been assumed to be zero in Eq.(5). A typical optical fiber consists of a core and a cladding fabricated from glasses which have similar properties. Hence, the fiber may be considered homogeneous and isotropic leading to

$$
p_{i j}=\left[\begin{array}{l}
p_{11} p_{12} \\
p_{12} p_{11}
\end{array}\right]
$$

Thus, the $\Delta \mathrm{n} l$ in the Eq. (2) for the light propagating in $\mathrm{z}$ direction ${ }^{[6]}$ is

$$
\left.\Delta n_{z}=-\frac{n^{2}}{2} *\left[\left(p_{11}+p_{12}\right) * \varepsilon_{r}+p_{12} * \varepsilon_{z}\right)\right]
$$

The third term in Eq. (2) can be shown to be negligible compared with the first two. A pressure $\mathrm{P}$ interacting with the fiber induces a change in phase $\Delta \square / \mathrm{P}$ and is given by

$$
\frac{\Delta \phi}{P}=k_{0} n_{0} \Delta L+k_{0} L \Delta n_{0}
$$

Where the first term corresponds to the change in the length of the fiber and the second term corresponds to the photoelastic effect. Therefore, the pressure sensitivity of the mandrel sensor per unit of air pressure becomes:

$$
\frac{\Delta \Phi}{\Phi}=\varepsilon_{z}-\frac{n^{2}}{2}\left[\left(P_{11}+P_{12}\right) \varepsilon_{r}+P_{12} \varepsilon_{z}\right] \ldots \ldots \ldots . . .(8)
$$

Equation (8) means that the phase change of the mandrel sensor can be found once we determine the appropriate strain distribution in relation to the unit of applied pressure, which then leads to the analysis of the transducer performance. In this paper, the strain distribution is calculated by using the MATLAB. Eq. (8) is written in the form of a summation of the strains distributed over all the discrete elements in the MATLAB. The following values for Pockel's coefficients of the unclad fiber are assumed in the calculations of the pressure sensitivity with the MATLAB ${ }^{[2]}$.

\section{Mathematical analysis of MZI}

In this paper a mathematical analysis of interferometer has to be done through MATLAB. Here after parameter like phase, frequency for MZI and fiber optical cable related parameter like core-cladding radius, length shows in the following graph simulated by MATLAB gives the pressure sensitivity. Interferometric arms differ by virtue of the perturbation in one of the fiber legs, and the phase shift between the two light signals provides the measurement. Because the measurements are made with the interference of two light signals with a small wavelength (typically $850-1550 \mathrm{~nm}$ ) the level of sensitivity is extremely high. In Fig. 2 Change in length Vs Pressure, analysis indicating pressure increases with changes in $\Delta \mathrm{l}$ w.r.t length of the fiber wrapped around the mandrel ' $\mathrm{L}$ ' will be less. Mathematical analysis of interferometer, and keeps a promise to give accuracy in the measurement of all the parameter and higher possibility of sensitivity. 


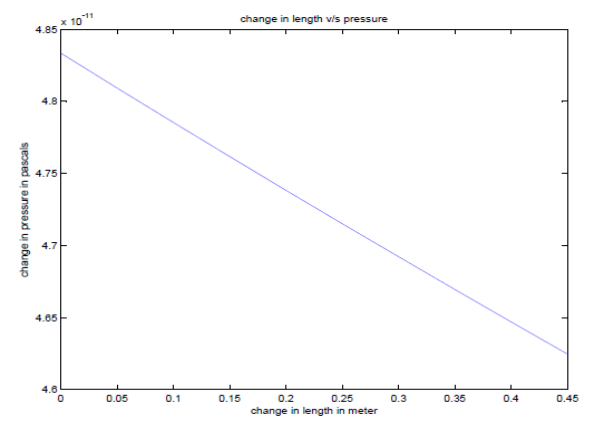

(a)

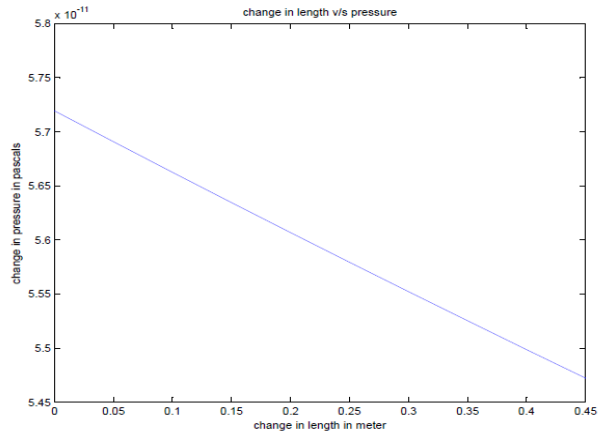

(b)

Fig2. Change in Length Vs Pressure

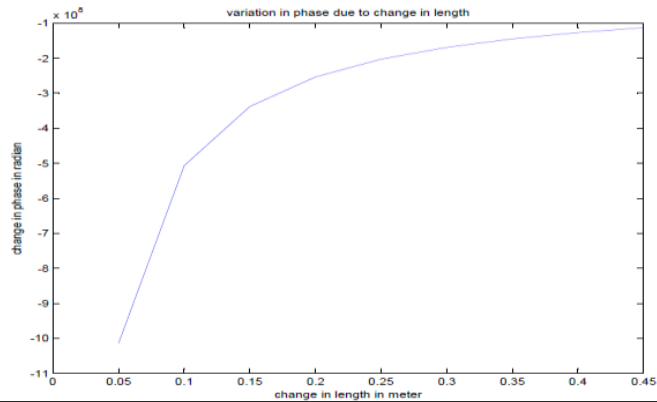

Fig3. Variation in phase due to change in length

From Fig 4 another parameter also keeping the promises of more sensitive like pressure (transverse load) occurs with the change in phase.

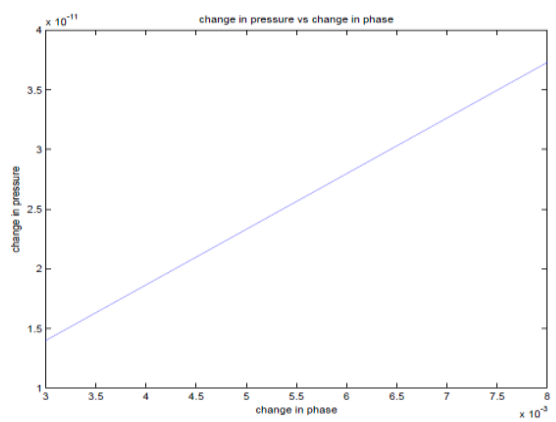

(a)

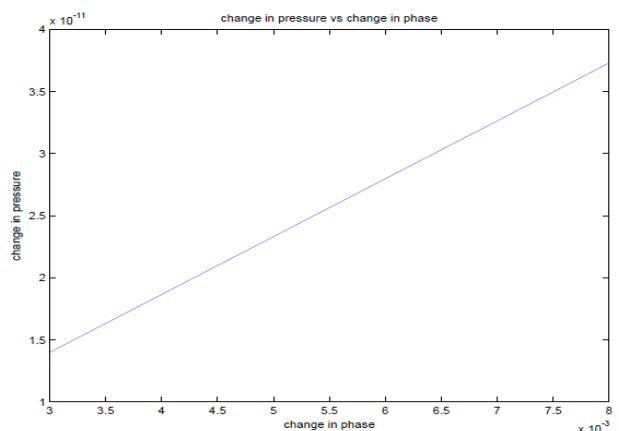

(b)

Fig.4 Change in pressure Vs Change in Phase

Radial transverse load applied to the mandrel, slightly increases diameter of the mandrel which in comparison with- without transverse load applied on mandrel of diameter is very less quantity of measurand, shown in Fig 5 .

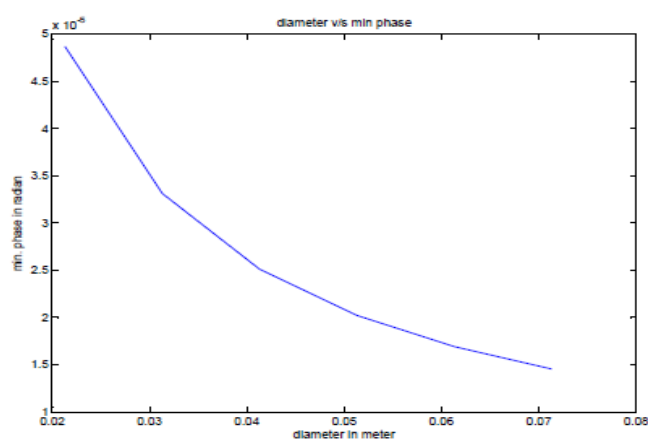

Fig 5 Diameter Vs Minimum Phase

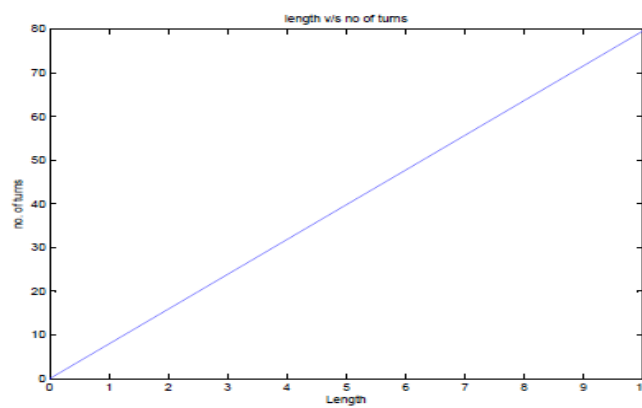

Fig.6 Length Vs Number of turns

Wrapping of the fiber on mandrel where it needed to find out 'No of Turns'. The reason is reducing bending losses. Fig 6 analyzing this as length increases, no of turn increases. From all above mentioned the 
graph shows: All the parameter has to verify with the different wavelength with different parameter and some of the graph mentioned here, length, no of turns, diameter, and min phase are showing the result that MZI interferometer sensor is more sensitive.

Following measurements are formed in table which gives the highest possibility of detecting the transverse load.

Table 1

\begin{tabular}{|c|c|c|c|c|c|}
\hline & Core & Clad & $\begin{array}{l}\text { First } \\
\text { coating } \\
\text { (soft) }\end{array}$ & $\begin{array}{l}\text { Second } \\
\text { coating } \\
\text { (soft) }\end{array}$ & $\begin{array}{l}\text { Polymer } \\
\text { layer } \\
\text { (Thick) }\end{array}$ \\
\hline Composition & $\mathrm{SiO} 2$ & $\begin{array}{l}95 \% \mathrm{SiO}_{2}, \\
5 \% \mathrm{~B}_{2} \mathrm{O}_{3}\end{array}$ & $\begin{array}{c}\text { Silicone } \\
\text { rubber }\end{array}$ & $\begin{array}{l}\text { Rimplast } \\
\text { Nylon }\end{array}$ & $\begin{array}{c}\text { polyuretha } \\
\text { ne }\end{array}$ \\
\hline Diameter $(\square \mathbf{m})$ & 10 & 125 & 220 & 300 & 1000 \\
\hline $\begin{array}{ll}\text { Young's } & \text { modulus } \\
\left(10^{10} \text { dyn } / \mathrm{cm}^{2}\right) & \end{array}$ & 72.5 & 72 & 0.0035 & 2.14 & 0.794 \\
\hline Poisson's ratio & 0.17 & 0.168 & 0.4994 & 0.44 & 0.45 \\
\hline $\mathbf{P}_{11}$ & 0.126 & & & & \\
\hline $\mathbf{P}_{12}$ & 0.27 & & & & \\
\hline $\mathbf{n}$ & 1.45 & $1 . .46$ & & & \\
\hline
\end{tabular}

IV. Photo Multiplier Tube

Light detection technology is a powerful tool that provides better understanding for more sophisticated phenomenon. Photodetector or light sensors are divided broadly in three categories: external photoelectric effect, internal photoelectric effect and thermal types. The external photoelectric effect is a phenomenon in which light strikes a semiconductor placed in vacuum, electrons are emitted from its surface into the vacuum. Photomultiplier Tube (PMT) makes use of detector as external photoelectric effect and is superior in response speed and sensitivity (low level light detection). Block diagram of MZI sensor, one arm of which (sensing mandrel arm) contains a fiber optic coil deform in an underwater sound pressure field. Since detectable acoustic pressure produce variations in the optical path length of the sensing mandrel. Transduction mechanism of such devices is directly dependent on the acoustically/ induced phase modulation of the sensing mandrel. After modulation of the light path from the sensing mandrel and reference mandrel both are combined and sent to a photomultiplier tube for detection.

Working Block Diagram

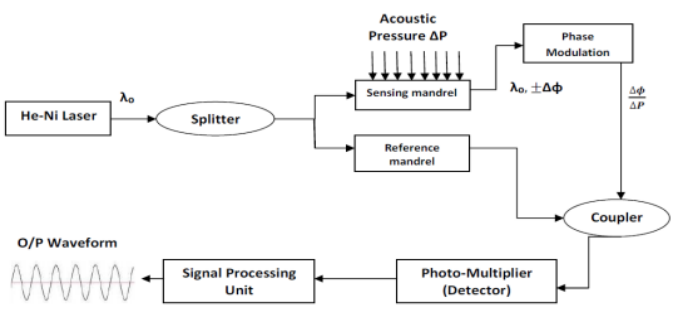

If two light signals are adjusted so that they are $90^{\circ}$ out of phase with each other, then the signal of the output current $i_{s}$ of the photomultiplier is ${ }^{[4]}$

$$
i_{s}=2 \beta S_{c} G\left(P_{R} P_{S}\right)^{1 / 2} \Delta \phi \ldots . .(9)
$$

Where $\beta$ is the homodyne efficiency, $S_{c}$ the photocathode sensitivity, $G$ the photomultiplier gain, $P_{R}$ and $P_{s}$ is the respective powers of the reference signal and sensing signal light, and $\Delta \square$ the acoustically/induced phase difference between two arms. For a borosilicate crown glass, $\Delta \square$ is evaluated to yield ${ }^{[4,9]}$

$$
i_{s}=8.1 * 10^{-18} \beta S_{c} G k_{0} l\left(P_{R} P_{S}\right)^{1 / 2} P \ldots \ldots \text { (10) }
$$

Where $k_{0}$ is free space optical wavenumber, $l$ is the length of the wrapped fiber around the mandrel, and $P$ is he applied acoustic pressure (in units of $\mu \mathrm{Pa}$ ). shown in the below fig.7 


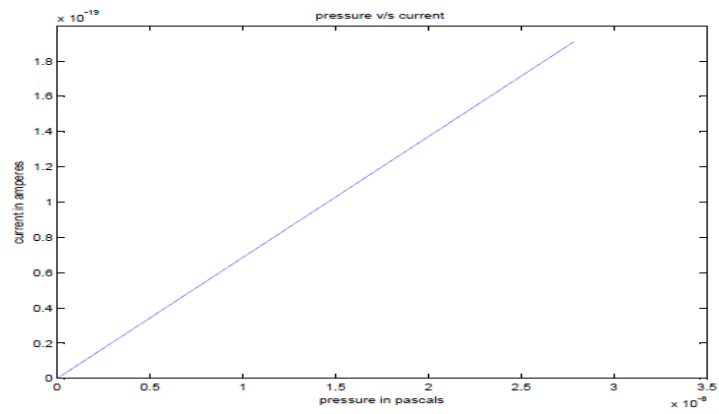

Fig.7 - Pressure Vs Current

\section{Conclusion}

In this paper, various parameters such as length, radius of mandrel, frequency of mandrel for transverse load sensitivity like pressure, increases the highest possible value of sensitivity of optical fiber based on interferometer principle - As Pressure increases, changes in light path i.e .phase modulation will occurs depending on the transverse load in the sensor arm which in comparisons with reference arm will be more so, the effective changes in the fiber length $(\Delta \mathrm{l})$ will be less and vice-versa. In this paper, we concluded, pressure increases with changes in $\Delta \mathrm{l}$ w.r.t length of the fiber wrapped around the mandrel ' $\mathrm{L}$ ' will be less.

Table 2

\begin{tabular}{|c|c|c|}
\hline $\begin{array}{c}\text { Length }(\Delta \mathrm{l}) \\
\text { In meter }\end{array}$ & $\begin{array}{c}\text { Pressure }(\Delta \mathrm{P}) \\
\text { In Pascal }\end{array}$ & $\begin{array}{c}\text { Phase }(\Delta \square) \\
\text { In Radian }\end{array}$ \\
\hline 0.05 & $4.8^{*} 10^{\wedge}-11$ & $7.5^{*} 10^{\wedge}-4$ \\
\hline 0.1 & $4.78^{*} 10^{\wedge}-11$ & $7^{*} 10^{\wedge}-4$ \\
\hline 0.15 & $4.7^{*} 10^{\wedge}-11$ & $6.5^{*} 10^{\wedge}-4$ \\
\hline
\end{tabular}

Table 2 shows the changes in the effective length $(\Delta \mathrm{l})=0.15$ at pressure of $4.7^{*} 10^{\wedge}-11$. Also, variation in phase of light path at same effective length observed is $6.5^{*} 10^{\wedge}-4$. All above graph of "Length Vs No of Turns, Diameter Vs Minimum Phase, Change in pressure Vs Change in Phase, Variation in phase due to change in length, Change in Length Vs Pressure", At photomultiplier tube, output current has been observed for borosilicate crown glass is $1.68^{*} 10^{\wedge}-19$ Ampere at pressure of $2.5 \mathrm{Mpa}$. Studies are promising the MZI interferometric sensor is more sensitive from other mechanically structure like strain-gauge, diaphragm.

\section{Reference}

[1] J. A. Bucaro, N. Lagakos, J. H. Cole, and T. G. Giallorenzi, "Fiber optic acoustic transduction," Physical Acoustics edited by R. H. Thurston, 16, 385 (1982)

[2] N. Lagakos, E. U. Schnaus, J. H. Cole, J. Jarzynski, and J. A. Bucaro, "Optimizing fiber coatings for interferometric acoustic sensors," IEEE Journal of Quantum Electronics, 18(4), 683 (1982)

[3] J. Jarzynski, R. Hughes, T. R. Hickman, J. A. Bucaro, "Frequency response of interferometric fiber optic coil hydrophone," Journal of Acoustical Society of America, 69(6), 1799 (1981)

[4] Peter Shajenko, James P. Flatley, and Mark B. Moffett, "On fiber hydrophone sensitivity" Naval Underwater system Center, New London Laboratory, New London, Connecticut 06320.

[5] R. Hughes and J. Jarzynski, "Static pressure sensitivity amplification in interferometric fiber optic hydrophones," Applied Optics, 19(1), 1 (1 9800$)$

[6] V. S. Sudarshanam and K. Srinivasan, "Static phase change in a fiber optic coil hydrophone," Applied Optics, 29(6), 855 (1990)

[7] J. F. Nye, "Physical Properties of Crystals,"(Clarendon, Oxford

[8] Press, 1976)

[9] G. B. Hocker, "Fiber Optic sensing of pressure and temperature" Applied Optics, 18(9), 1445 (1979).

[10] G. A. Massey, “An Optical heterodyne Ultrasonic Image Converter” Proc. IEEE 56, 2157-2161 (1968). 